\title{
Spin-charge-orbital ordering on triangle-based lattices
}

\author{
Hiroaki Onishi* , Takashi Hotta \\ Advanced Science Research Center, Japan Atomic Energy Research Institute, Tokai, Ibaraki 319-1195, Japan
}

\begin{abstract}
We investigate the ground-state property of an $e_{\mathrm{g}}$-orbital Hubbard model at quarter filling on a zigzag chain by exploiting the density matrix renormalization group method. When two orbitals are degenerate, the zigzag chain is decoupled to a doble-chain spin system to suppress the spin frustration due to the spatial anisotropy of the occupied orbital. On the other hand, when the level splitting is increased and the orbital anisotropy disappears, a characteristic change in the spin incommnsurability is observed due to the revival of the spin frustration.
\end{abstract}

Key words: Geometrical frustration, Orbital ordering, Spin incommensurability, Density matrix renormalization group method PACS: 75.10.-b; 71.10.Fd; 75.30.Et

The magnetic property of frustrated spin systems has been one of the central issues for many years in the research field of condensed matter physics [1]. For example, it is well known that in the antiferromagnetic (AFM) Ising model on a triangular lattice, there occurs macroscopic degeneracy for possible spin configurations in the ground state [2]. In general, however, such high degeneracy is lifted to suppress the spin frustration, since the lattice is deformed to lower the lattice symmetry due to the spin-lattice coupling.

On the other hand, recently there has been a rapid increase of interest in the effect of the interplay of spin and orbital degrees of freedom [3]. It has been emphasized that the orbital anisotropy plays a significant role to cause a variety of cooperative phenomena in realistic materials. In particular, in geometrically frustrated lattices, it is expected that orbital ordering occurs to affect the spin frustration due to the spin-orbital coupling, since the orbital anisotropy leads to the nonuniform exchange interactions.

In this paper, to clarify the key role of the orbital anisotropy in geometrically frustrated lattices, we investigate an $e_{\mathrm{g}}$-orbital Hubbard model on a zigzag chain with one electron per site (quarter filling). When

\footnotetext{
* Corresponding author.

Email address: onishi@season.tokai.jaeri.go.jp (H. Onishi)
}

the Hund's rule coupling $J$ is small, the ground state is found to be paramagnetic (PM) [4,5], which is relevant to a geometrically frustrated antiferromagnet. Here we study the property of the PM phase and set $J=0$ for simplicity. The effect of $J$ has been investigated for an $e_{\mathrm{g}}$-orbital degenerate model [5].

The Hamiltonian considered here is given by

$$
\begin{aligned}
H= & \sum_{\mathbf{i}, \mathbf{a}, \gamma, \gamma^{\prime}, \sigma} t_{\gamma \gamma^{\prime}}^{\mathbf{a}} d_{\mathbf{i} \gamma \sigma}^{\dagger} d_{\mathbf{i}+\mathbf{a} \gamma^{\prime} \sigma}-(\Delta / 2) \sum_{\mathbf{i}}\left(\rho_{\mathbf{i} a}-\rho_{\mathbf{i} b}\right) \\
& +U \sum_{\mathbf{i}, \gamma} \rho_{\mathbf{i} \gamma \uparrow} \rho_{\mathbf{i} \gamma \downarrow}+U^{\prime} \sum_{\mathbf{i}} \rho_{\mathbf{i} a} \rho_{\mathbf{i} b}
\end{aligned}
$$

where $d_{\mathbf{i} a \sigma}\left(d_{\mathbf{i} b \sigma}\right)$ is the annihilation operator for an electron with spin $\sigma$ in the $3 z^{2}-r^{2}\left(x^{2}-y^{2}\right)$ orbital at site $\mathbf{i}, \rho_{\mathbf{i} \gamma \sigma}=d_{\mathbf{i} \gamma \sigma}^{\dagger} d_{\mathbf{i} \gamma \sigma}$, and $\rho_{\mathbf{i} \gamma}=\sum_{\sigma} \rho_{\mathbf{i} \gamma \sigma} . t_{\gamma, \gamma^{\prime}}^{\mathbf{a}}$ is nearestneighbor hopping between $\gamma$ and $\gamma^{\prime}$ orbitals along the $\mathbf{a}$ direction. Note that the zigzag chain is considered as a double chain connected by a zigzag path. The hopping amplitudes are given by $t_{a a}^{\mathbf{x}}=t / 4, t_{a b}^{\mathbf{x}}=t_{b a}^{\mathbf{x}}=-\sqrt{3} t / 4$, $t_{b b}^{\mathbf{x}}=3 t / 4$ for the double-chain direction and $t_{a a}^{\mathbf{u}}=t / 4$, $t_{a b}^{\mathbf{u}}=t_{b a}^{\mathbf{u}}=\sqrt{3} t / 8, t_{b b}^{\mathbf{u}}=3 t / 16$ along the zigzag path. Hereafter, $t$ is taken as the energy unit. $\Delta$ is the level splitting between $3 z^{2}-r^{2}$ and $x^{2}-y^{2}$ orbitals, $U$ is the intraorbital Coulomb interaction, and $U^{\prime}$ is the interorbital Coulomb interaction. 
(a)
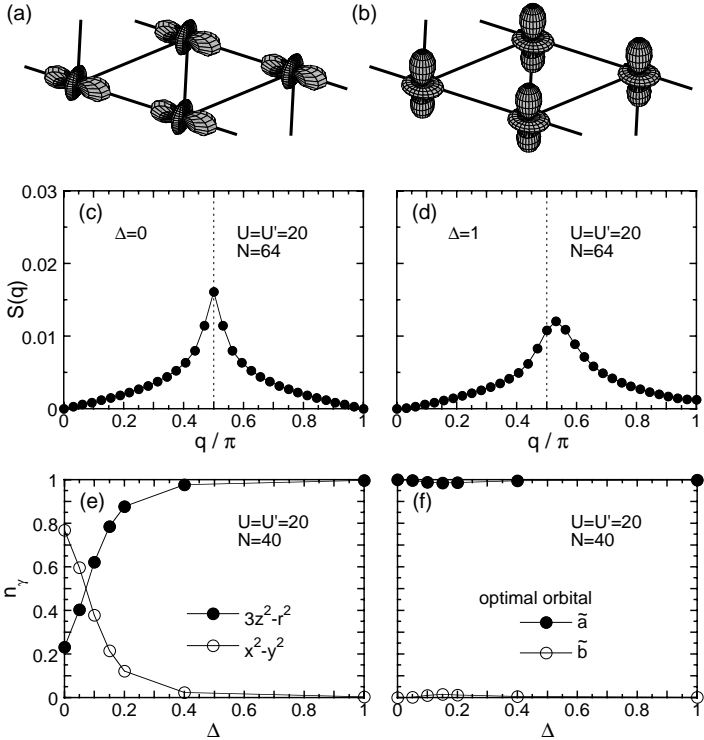

Fig. 1. Orbital structure for (a) $\Delta=0$ and (b) $\Delta=1$ from the DMRG results for $N=40$ at $U^{\prime}=U=20$. Spin correlation for (c) $\Delta=0$ and (d) $\Delta=1$. Electron densities in (e) $3 z^{2}-r^{2}$ and $x^{2}-r^{2}$ orbitals and (f) optimal $\tilde{a}$ and $\tilde{b}$ orbitals.

We analyze the model with $N$ sites in the open boundary condition by using the density matrix renormalization group (DMRG) method [6]. To reduce the size of the superblock Hilbert space, we treat each orbital as a site. We employ the finite-system algorithm with keeping up to 200 states per block and the truncation error is estimated to be $4 \times 10^{-6}$ at most.

In order to determine the orbital structure, we introduce new operators by using an angle $\theta_{\mathbf{i}}$ such as $\tilde{d}_{\mathbf{i} \tilde{a} \sigma}=e^{i \theta_{\mathbf{i}} / 2}\left[\cos \left(\theta_{\mathbf{i}} / 2\right) d_{\mathbf{i} a \sigma}+\sin \left(\theta_{\mathbf{i}} / 2\right) d_{\mathbf{i} b \sigma}\right]$ and $\tilde{d}_{\mathbf{i} \tilde{b} \sigma}=$ $e^{i \theta_{\mathbf{i}} / 2}\left[-\sin \left(\theta_{\mathbf{i}} / 2\right) d_{\mathbf{i} a \sigma}+\cos \left(\theta_{\mathbf{i}} / 2\right) d_{\mathbf{i} b \sigma}\right]$ [7]. The optimal orbitals, $\tilde{a}$ and $\tilde{b}$, are determined so as to maximize the orbital correlation $T(\mathbf{q})=\sum_{\mathbf{i}, \mathbf{j}} e^{i \mathbf{q} \cdot(\mathbf{i}-\mathbf{j})}\left\langle\tilde{T}_{\mathbf{i}}^{z} \tilde{T}_{\mathbf{j}}^{z}\right\rangle / N^{2}$ with $\tilde{T}_{\mathbf{i}}^{z}=\sum_{\sigma}\left(\tilde{d}_{\mathbf{i} \tilde{a} \sigma}^{\dagger} \tilde{d}_{\mathbf{i} \tilde{a} \sigma}-\tilde{d}_{\mathbf{i} \tilde{\mathbf{i}} \sigma}^{\dagger} \tilde{d}_{\mathbf{i} \tilde{b} \sigma}\right) / 2$. In Figs. 1(a) and (b), the orbital structure is shown for $\Delta=0$ and 1 , respectively. When two orbitals are degenerate for $\Delta=0$, orbital degree of freedom is active, but a $3 x^{2}-r^{2}$ orbital is selectively occupied to suppress the spin frustration, as shown in Fig. 1(a). Namely, the orbital shape extends just along the double-chain direction, and the zigzag chain is decoupled to a double-chain spin system due to the orbital anisotropy. In fact, the ratio of the AFM exchange interaction along the double-chain direction $J_{2}$ to that along the zigzag path $J_{1}$ is estimated as $J_{2} / J_{1}=64^{2}$. On the other hand, for $\Delta=1$, a lower-energy $3 z^{2}-r^{2}$ orbital is favorably occupied, as shown in Fig. 1(b). Note that when the $3 z^{2}-r^{2}$ orbital is fully occupied for infinite $\Delta$, the orbital anisotropy disappears in the $x y$ plane, i.e., $J_{2} / J_{1}=1$, and the spin frustration becomes effective.

In accordance with the variation in the orbital shape, the spin state is also changed. To clarify this point, it is convenient to reduce the present model to a spin system on the orbital-ordered background. Then, the present system is described by the zigzag spin chain, in which the spin correlation has a commensurate peak at $q=\pi$ for $0 \leq J_{2} / J_{1} \leq 1 / 2$, but the peak is gradually changed to an incommensurate one for $J_{2} / J_{1} \geq 1 / 2$, and eventually, we find the incommensurate peak at $q=\pi / 2$ for infinite $J_{2} / J_{1}[8,9]$. In Fig. 1(c), we show our DMRG result of the spin correlation $S(\mathbf{q})=\sum_{\mathbf{i}, \mathbf{j}} e^{i \mathbf{q} \cdot(\mathbf{i}-\mathbf{j})}\left\langle S_{\mathbf{i}}^{z} S_{\mathbf{j}}^{z}\right\rangle / N^{2}$ with $S_{\mathbf{i}}^{z}=\sum_{\gamma}\left(\rho_{\mathbf{i} \gamma \uparrow}-\rho_{\mathbf{i} \gamma \downarrow}\right) / 2$ for $\Delta=0$. We find a clear peak at $q=\pi / 2$, consistent with that of the zigzag spin chain with large $J_{2} / J_{1}$, since $J_{2} / J_{1}=64^{2}$ for $\Delta=0$. On the other hand, as shown in Fig. 1(d), the peak position for $\Delta=1$ changes from $q=\pi / 2$ toward $q=\pi$, expected by analogy with the zigzag spin chain, since we estimate $J_{2} / J_{1}=1.61$ for $\Delta=1$. The detail of the $\Delta$ dependence will be discussed elsewhere in future.

Finally, let us consider how the orbital state changes in the intermediate region. In Fig. 1(e), we show the $\Delta$ dependence of the electron densities $n_{\gamma}=\sum_{\mathbf{i}}\left\langle\rho_{\mathbf{i} \gamma}\right\rangle / N$ in $3 z^{2}-r^{2}$ and $x^{2}-r^{2}$ orbitals. With increasing $\Delta$, electrons are forced to accommodate in the lower $3 z^{2}-r^{2}$ level, but the electron density in each orbital is found to change gradually without any singularity. To understand this behavior, we evaluate the electron densities for the optimal orbitals. As shown in Fig. 1(f), it is found that one of the optimal orbitals is occupied irrespective of $\Delta$ and the fluctuation is very small even in the intermediate region. Namely, the present system is always regarded as a one-orbital system, although we have considered the multi-orbital system.

In summary, for $\Delta=0$, the $3 x^{2}-r^{2}$ orbital is selectively occupied to suppress the spin frustration and the zigzag chain is decoupled to a double chain due to the orbital anisotropy. For large $\Delta$, the $3 z^{2}-r^{2}$ orbital is occupied and the spin frustration revives, leading to the change in the spin commensurability.

T.H. is supported by the Japan Society for the Promotion of Science and by the Ministry of Education, Culture, Sports, Science, and Technology of Japan.

\section{References}

[1] H. T. Diep, (Ed.), Frustrated Spin Systems, World Scientific, Singapore, 2004.

[2] G. H. Wannier, Phys. Rev. 79 (1950) 357.

[3] E. Dagotto et al., Phys. Rep. 344, 1 (2001).

[4] H. Onishi, T. Hotta, Physica B 359-361 (2005) 669.

[5] H. Onishi, T. Hotta, Phys. Rev. B 71 (2005) 180410(R).

[6] U. Schollwöck, Rev. Mod. Phys. 77 (2005) 259.

[7] T. Hotta et al., Int. J. Mod. Phys. B 12 (1998) 3437.

[8] T. Tonegawa, I. Harada, J. Phys. Soc. Jpn. 56 (1987) 2153.

[9] S. R. White, I. Affleck, Phys. Rev. B 54 (1996) 9862. 